
\title{
Reseracth Sulure \\ The Effect of Balloon Inflating Distraction on Pain Intensity During Venous Blood Sampling in Children: A Randomized Controlled Trial
}

Somaeh. Salehi Sarbijan

Kerman University of Medical Sciences

Roghiah Mehdipoor Rabori

Kerman University of Medical Sciences

Esmat Nouhi (D e_nuhi@kmu.ac.ir)

Kerman University of Medical Sciences

\section{Research Article}

Keywords: pain in children, venous blood sampling, inflating balloons

Posted Date: August 11th, 2021

DOI: https://doi.org/10.21203/rs.3.rs-711305/v1

License: (1) (i) This work is licensed under a Creative Commons Attribution 4.0 International License.

Read Full License 


\title{
The effect of balloon inflating distraction on pain intensity during venous blood sampling in children: A randomized controlled trial
}

Running title: Effect of Balloon Inflating on Pain Level during the Venous Blood Sampling in Children

\author{
Somaeh. Salehi Sarbijan"1, Roghiah Mehdipoor Rabori ${ }^{2,3}$, Esmat Nouhi4,5 \\ 1. M.s. Neonatal Nursing, Nursing Research Center, Kerman University of Medical \\ Sciences, Kerman, Iran \\ Email: ssina8650@gmail.com
}

2. Nursing Research Center, Kerman University of Medical Sciences, Kerman, Iran.

3. Razi Faculty of Nursing and Midwifery, Department of Medical Surgical Nursing, Kerman University of Medical Sciences, Kerman, Iran.

Email:rm41321@yahoo.com

ORCID ID: 0000-0002-7608-9545

4. Razi Faculty of Nursing and Midwifery, Department of Medical Surgical Nursing, Kerman University Of Medical Sciences, Kerman, Iran

5. Nursing Research Center, Kerman University of Medical Sciences, Kerman, Iran.

ORCID ID: 0000-0003-1906-4146

Email: e_nuhi@kmu.ac.ir (Corresponding Author)

Telephone number: 09133407727 


\begin{abstract}
Background: A large number of children are subject to prevention, diagnosis, and treatment procedures that mostly require venous blood sampling. Painful procedures such as blood sampling in children have annoying physical and emotional effects and can lead to wider negative consequences such as physical, behavioral, and social disorders in children. This study aimed to determine the effect of distraction by balloon inflating on the amount of pain during taking blood samples from children aged 4 to 7.
\end{abstract}

Method: This study had a randomized controlled clinical trial that was conducted on 80 children admitted to the pediatric department of Imam Khomeini Hospital in Jiroft- Kerman province who met the inclusion criteria after obtaining their parent's consent. The children were randomly grouped into the intervention $(n=40)$ and control $(n=40)$ groups. The pain level in children in the intervention group was measured during the venous blood sampling while the children were watching inflating a balloon. However, the pain in children in the control group was measured without any intervention during blood sampling. Wong-Baker Faces Pain Rating Scale questionnaire was used for pain assessment.

Results: The average pain scores for the children in the two groups showed no significant difference before the intervention but the average pain scores after the intervention in the intervention and control groups were $2.56 \pm 1.38$ and 4.2 \pm 1.58 ; showing a significant difference between the two groups after the intervention ( $\mathrm{p}$-value $<0.05)$.

Conclusion: Using balloon inflation distraction during venous blood sampling reduces pain in children.

Keywords: pain in children, venous blood sampling, inflating balloons 


\section{Introduction}

Pain is a mental feeling and reaction caused by the perception of the nerves on different parts of the body against the internal or external stimuli and it is one of the most common symptoms of physical and emotional discomfort experienced by a person[1]. Pain can lead to physiological changes such as raising heart rate and breathing, sweating, skin redness, reducing blood oxygen saturation, dilated pupils, restlessness, and hypertension [2]. Pain is one of the most important defense and protection mechanisms in the body that appears under abnormal conditions [3]. Because of the importance of pain and its control, the Pain American Academy has ranked pain as the fifth vital sign and states that whenever pain is controlled as seriously as other vital signs are monitored, the treatment team will be able to provide better drug and non-drug treatments[4]. Children are not alien to pain and experience it since their first days of life by blood sampling and vaccination. Besides, during childhood, they feel it by common childhood disease or painful accidents [5]. Pain is defined as one of the health problems in children. Hospitalized children undergo painful procedures. Most children consider such procedures as the most stressful events and one of the most damaging aspects of hospitalization. In response, they cry and feel scared in these situations and try to refuse them [3]. Following the frequent experience of pain, permanent changes such as autonomic nervous system stimulation occurs that can disrupt neurodevelopment and learning ability and lead to behavioral problems in children. Children's response to pain is influenced by the interaction of factors such as genetics, experience, and evolutionary factors, not to mention the role of private life experience that results in differences in response. These components increase response to pain potentially. Thus, medical staff should pay attention to their important role in reducing pain [1]. One of the functions of nurses in protecting patients' rights is to control and reduce pain especially in children and try to alleviate anxiety caused by hospitalization $[6,7]$. On the other hand, if the pain is not controlled, the child's negative response and mental suffering lead to more problems and less successful treatment [3]. Furthermore, the unnecessary pain leads to the child's lack of confidence in nurses and care providers. This, in turn, may make the child less willing to undergo remedial measures. Moreover, inadequate pain relief in children leads to undesirable immediate and long-term consequences[8]. Pain that is not relieved enough increases child's anxiety during the treatment 
procedure and will have undesirable physiological consequences. It will also increase fear and avoidance of medical procedures [8]. Other harmful effects of pain on children's lives include the child's impaired performance, failure to perform the family and social roles, retrogression to the past development stages, and treatment phobia. What's more, the negative memory of painful treatment in younger children leads to negative perceptions of clinical care [1,9]. The American Academy of Pediatrics and the American Pain Society suggest reducing stress and pain to a minimum level in treatment procedures [4].

The Agency for Health Care Policy and Research (AHCPR) states that the effective treatment of pain includes the use of drug and non-drug methods. The most important non-drug pain relief intervention is behavior therapy which uses distraction techniques. In this method, the individual is distracted from painful stimuli and attracted to a pleasant one which reduces pain perception. In addition to less or no complications compared to drug-oriented methods, this method is less costly. Moreover, it has no devastating physical and psychological effects on children. It is also easily applicable and does not require much training. Distraction does not require a lot of time for implementation and more importantly, it is among the independent nursing actions that are attractive for young children [10]. Therefore to reduce the psychological and physical effects of painful interventions and to prevent the long-term effects of pain in children, nurses should be able to handle painful procedures [11]. Audio techniques such as music and audio-visual techniques and television are used as the most common types of passive distraction for sick children [12]. Various distraction methods are used for pain relief in children. For example, Gupta et al. studied reducing pain associated with venous opening using balloon inflating distraction and the results presented a statistically significant difference between the group with distraction techniques and the group without any pain relief measure [6]. Kleiber et al. studied the amount of pain and anxiety caused by venous opening in 4-7-year-old children using a selfreport tool. They used the distraction technique in the intervention group and their results showed that there was a statistically significant intergroup difference in terms of pain and anxiety [13]. Caprilli et al. examined the effect of music on children's distress and pain during blood sampling and found that pain intensity was significantly lower in the test group than in the control group [14]. Wang et al. conducted a study on the school-age children who were hospitalized in the pediatric department and were subject to venipuncture in China and conducted audiovisual distraction in the intervention group. The results indicated a significant 
difference between the intervention and control groups in terms of pain perception [15]. Mutlu et al. conducted a study on 9-12-year-old children who were hospitalized in Istanbul and were subject to blood sampling. They studied the effect of deep inhalation on venous blood sampling and found a statistically significant difference between the intervention and control groups[4].

Esmaili et al. examined the effectiveness of breathing exercises and music in reducing venipuncture pain during the blood transfusion and showed that the amount of pain was reduced due to the use of distraction and relaxation (practicing regular breathing and the use of music). However, music was more effective in reducing pain so that after completion of the study, the patients requested to use headphones during venipuncture [16]. Shahabi and Kalani studied the effect of topical local anesthetic ointment (EMLA) and distraction (music) on pain caused by venipuncture in school students in a hospital in Shiraz and found that the use of EMLA ointment and distraction significantly reduced pain during venipuncture [17]. Sadeghi investigated the effect of balloon inflating on pain caused by venous opening and reported lower levels of pain in the intervention group compared to the control group[18]. Mehdipour et al. studied the effect of distraction on venipuncture pain in children with strabismus and showed that the average pain intensity was not significantly different in the two distraction groups that used touching and play therapy but the pain level was significantly lower for these groups compared to the control group [3]. Razeghi) studied the effect of distraction (bubble maker) and touching on venipuncture pain in 5-10-year-old children and reported a statistically significant difference between the distraction (bubble maker) and touching and normal methods. However, there was no statistically significant difference between the distraction (bubble making) and touching methods [9]. Bagherian et al. studied the effect of distraction (rhythmic breathing and bubble making) on pain and anxiety caused by injection practices among the school-age children with thalassemia in Kerman. They reported that the distraction methods can reduce injection pain in children [19]. Rostami et al.) addressed the Hugo point ice massage on pain from venipuncture in children with thalassemia in Kerman and a statistically significant difference between the mean score of behavioral reactions caused by venipuncture in children in the intervention and control groups and pain was decreased in the intervention group [20].

According to the results of these studies and the fact that pain reduction in sick children is one of their rights, and nurses should use any right measure for their pain relief, this study was 
conducted to investigate the effect of distraction (balloon inflating) on pain caused by blood sampling in 4-7-year-old children.

\section{Methods}

\section{Study type and setting}

This study had a randomized controlled clinical trial design .This study was conducted as an intervention clinical trial with the registration number IRCT2016111329817N2 to explore the effect of balloon inflating intervention on pain during blood sampling in 4-7-year-old children hospitalized in the pediatric department of Khomeini Hospital in Jiroft- Kerman in 2016. The researchers obtained written consent from the parents and verbal consent from the children.

\section{Sample size and sampling}

Based on similar studies with the test power of $80 \%$, the significance level of 0.05 , maximum type I error 1.96, and type II error 1.28, the sample size was estimated as 30 patients for each group[9]. Considering the potential $10 \%$ dropout rate, the final sample size was considered 40 patients per group. (Figure 1).

\section{Data collection tool}

To measure pain, the Wong-Baker Faces Pain Rating Scale was administered to children. The scale is a visual pain grading scale and its validity and reliability were approved in previous studies[21]. In Iran, this tool has been used in many studies. Nikfarid reported its correlation coefficient as $82 \%[22,23]$.

\section{Intervention}

The researcher performed sampling and classified the children who met the inclusion criteria randomly or by throwing coins into the intervention or control groups. The inclusion criteria consisted of 4-7-year-old children, filling the written consent to participate in the study by the 
parents, the first reference for blood sampling, lack of known hypersensitivity to plastic materials in children, no specific disease in children (thyroid disorder, epilepsy, cardiovascular disease, and hyperactivity), and no specific illness or medication that would reduce the level of consciousness. Moreover, children who did not have clear arteries and the ones with a history of hospitalization were excluded [9]. To reduce the effect of confounding interventions, the needles, cotton, and alcohol with the same brands were used, blood sampling was performed by a skilled person between 7 and 9 a.m., all conditions were the same for both groups during the study, and all applied balloons were green (the green color has a calming effect in inflammatory conditions of the body, repairs the body cells, and it is also effective on the sympathetic nervous system)[24]

Before blood sampling, the children's pain level was measured by the Wong-Baker Faces Pain Rating Scale. In fact, children pointed to the image that was the most representative of their pain and the researcher marked it and then she talked to the children in the intervention group and showed them how to blow a balloon. She was assured that the children could blow a balloon and the necessary explanations about blood sampling were presented to them. In the intervention group, each child was given a balloon and was asked to blow it during the blood sampling (maximum 10 seconds). Blood sampling in the control group was conducted without any intervention. The elbow veins were used due to their visibility. A demographic information form (age, gender disease, birth order and etc.) was completed by the parents of the children in both groups and they were asked to stay by the child during blood sampling and during training. The researcher answered the questions asked by the parents and children.

\section{Data analysis}

SPSS 18, trial version, was used to analyze data. Descriptive statistics (frequency, percent, mean, and standard deviation) were used to describe the demographic and background characteristics of both groups. $t$ independent, Mann-Whitney $\mathrm{U}$ and $\chi 2$ tests were used to compare two groups.

\section{Ethical considerations}

This study was approved under the code of ethics IR.Kmu.REC.1390.260. Besides, the objectives of the study were explained to the parents. Informed consent was obtained from both 
parents and children. All procedures were performed by taking into account the participants' physical and mental comfort. The participants could leave the study at any time they desired.

\section{Results}

Of 80 patients who participated in the study, 40 patients were in the control group and 40 patients were in the intervention group. The children's age ranged from 4 to 7. A majority of the participants, 47 patients $(\approx 60 \%)$, were girl. No significant difference was observed between the two groups in terms of demographic variables. Table 1, 2 shows the most important demographic variables of the participants.

Table 3 shows that there was no significant difference in pain intensity before the intervention in control and test groups. However, there was a statistically significant difference after the intervention ( $\mathrm{p}$-value $<0.05$ ).

The results of the paired samples t-test indicated that the mean pain intensity scores in the control group before and after the intervention were $2.65 \pm 1.38$ and $4.2 \pm 1.5$, showing no statistically significant difference ( $\mathrm{p}$-value $>0.05$ ). In addition, the mean pain intensity scores in the test group before and after the intervention were $3.32 \pm 1.28$ and $2.78 \pm 1.18$, showing a statistically significant decrease in pain intensity after the intervention (p-value <0.05) (table3) .

\section{Discussion}

This study investigated the effect of distraction (balloon inflating) on pain severity in children during blood sampling. The data in this study showed that the mean pain score in the intervention group was lower than that of the control group indicating that balloon inflating distraction was effective in pain relief during blood sampling. Similarly, Abuelkheir et al. (2014) evaluated the effect of EMLA ointment on relieving pain caused by children's vaccination and reported higher pain in the control group [25]. Pourmovahed et al. (2008) compared the effects of EMLA ointment and music on pain intensity during venipuncture in children and showed that the use of drug and non-drug methods is effective in venipuncture pain relief. Besides, pain severity in the test group was lower than in the control group [26]. In a similar study, Nikfarid et 
al. examined the effectiveness of EMLA ointment and ice in reducing venipuncture pain in children hospitalized in the urology unit of Children's Medical Center and found that pain severity was lower in the test group[24]. Razeghi (2012) studied the effect of distraction and touching on venipuncture pain in 5 children and reported that bubble makers and touching were effective in reducing venipuncture pain in children [9]. Sadeghi used pain behavioral tools to assess pain and reported a higher level of pain in the control group [18].

All studies reviewed above indicated that pain is not relieved if no alleviation measure is taken. Moreover, the results of previous studies on the effectiveness of non-drug method in venipuncture pain relief were not consistent with this study. However, none of the above studies addressed pain intensity during blood sampling since blood sampling and venipuncture are invasive procedures that cause pain in children[19] and pain prevention and relief in children is very important[17]. Moreover, as the number of children who refer for blood sampling is greater than the ones referring for other procedures, it is important to use a method to relieve their pain. Nevertheless, studies have shown that nursing and laboratory personnel rarely use pain control methods. Thus, adequate training to use distraction alone to create an experience without fear and pain for children who undergo venipuncture and blood sampling is a necessary part of their treatment. These non-drug methods are important due to the low cost and availability compared to drug-based methods[24].

\section{Conclusion}

The results of this study highlighted the effectiveness of balloon inflation during venous blood sampling in reducing pain in children. Since balloon inflation is desired by children and it is a safe and low-cost treatment, it can be used as a game to relieve pain in children.

\section{Limitations}

There were some limitations in this study. First, providing inadequate or false responses to the questions by some parents, to solve this problem, the research purpose and significance was explained to the parents. Second, factors such as fatigue and mental engagement could affect the participants' responses to the questions. Thus, the questionnaires were completed at an appropriate time. The third limitation was the inability of some medical staff to carry out 
successful blood sampling. To solve this problem, blood samples were taken by skilled personnel.

\title{
Declarations
}

\begin{abstract}
Abbreviations
VBS: Venous Blood Sampling; AHCPR: Agency for Health Care Policy and Research; WBFPRS; Wong-Baker Faces Pain Rating Scale
\end{abstract}

\section{Ethics approval and consent to participate}

This project has been approved by the Ethics Committee of Kerman University of Medical Sciences with the ethics code IR.Kmu.REC.1390.260. In all stages of the research, the ethical principles mentioned in the 2013 edition of the Helsinki Statement were followed. The study's objectives were explained to the patients by the researcher and informed consent was obtained from all participants (patients and parents). The questionnaires were anonymous and were kept confidential, with scientific accuracy and reliability in recording the information and statistics obtained at the time of collection and analysis. The persons were free to participate in the study. All methods were carried out in accordance with relevant guidelines and regulations.

\section{Consent for Publication}

The information and statistics obtained were kept confidential at the time of collection and analysis. Images of identification or other personal or clinical characteristics of the participants were not provided in the results and report of the study, which makes the participants anonymous. Confidential release consent obtained from patients and nurses.

\section{Availability of data and material}

The datasets used and/or analyzed during the current study available from the corresponding author on reasonable request.

\section{Competing interests}


The authors declare that they have no competing interests.

\section{Funding}

This research project received no specific grant from any funding agency in the public, commercial, or not-for-profit sectors.

\section{Authors' contributions}

SS, EN, and RM participated in the design of the study. SS performed all the Phases of the modified systematic review. RM was involved in methodological assessment. SS wrote the initial draft of this paper and EN gave comments on all the subsequent and final version of the paper. RM and EN read and approved the final manuscript.

\section{Acknowledgments}

The authors would like to appreciate the cooperation of children and parents who participated in the study as well as the collaboration of the research council and the officials of Kerman University of Medical Sciences and staff in Khomeini Hospital of Jiroft.

\section{References}

1. Babaei M, Farahani A, Norian M, Hosaingholi A, Masompour A: Pain control methods of schoolage children through distraction. 2015, 10(3):71-80.

2. Wong DL, Hockenberry MJ, Wilson D: Wong's nursing care of infants and children. 2011.

3. mehdipour $R$, nematallahi $M$, esmaeilzadeh $F$ : The effect of distraction on pain severity in children with strabismus to vessel. Journal of nursing and midwifery, Hamadan, Iran 2011, 18(1):18-28.

4. Mutlu B, Balcı S: Effects of balloon inflation and cough trick methods on easing pain in children during the drawing of venous blood samples: A randomized controlled trial. Journal for Specialists in Pediatric Nursing 2015, 20(3):178-186.

5. Namnabati $M$, Abazari $P$, Talakob S: The experiences of nurses in pediatric pain management. Nursing Research 2008, 3(10):75-86.

6. Gupta D, Agarwal A, Dhiraaj S, Tandon M, Kumar M, Singh RS, Singh PK, Singh U: An evaluation of efficacy of balloon inflation on venous cannulation pain in children: a prospective, randomized, controlled study. Anesthesia \& Analgesia 2006, 102(5):1372-1375. 
7. nohi $E$, karbalaeizadeh $M$, abazari $F$ : The effect of family-centered care and participation on anxiety of mothers of children with gastrointestinal infections. Journal of clinical nursing and midwifery 2014, 3(4):47-55.

8. Agosto C, Farina MI, Catalano I, Coccato F, Lazzarin P, Benini F: Procedural pain in children: education and management. The approach of an Italian pediatric pain center. European journal of pediatrics 2012, 171(8):1175-1183.

9. Razeghi N, Gevari A, Tatarpor P, Hosaini F: Comparison of the effect of distraction and touch on the intensity of the pain caused by the vessel-making in children aged 5-10. Journal of nursing 2012, 25(77):50-59.

10. Alavi A, Namnabati M, Abdyazdan Z, Parvin N, Akbari N, Samepor V, Daris F: How to manage children's pain by the nursing staff in the hospitals of the city of shahrekord, in 1385. Shahrekord University of medical sciences journal 2008, 10(2):59-65.

11. Hasanpour M, Tootoonchi M, Aein F, Yadegarfar G: The effects of two non-pharmacologic pain management methods for intramuscular injection pain in children. Acute pain 2006, 8(1):7-12.

12. Koller D, Goldman RD: Distraction techniques for children undergoing procedures: a critical review of pediatric research. Journal of pediatric nursing 2012, 27(6):652-681.

13. Kleiber C, Craft-Rosenberg M, Harper DC: Parents as distraction coaches during IV insertion: $\mathbf{A}$ randomized study. Journal of pain and symptom management 2001, 22(4):851-861.

14. Caprilli S, Anastasi F, Grotto RPL, Abeti MS, Messeri A: Interactive music as a treatment for pain and stress in children during venipuncture: a randomized prospective study. Journal of Developmental \& Behavioral Pediatrics 2007, 28(5):399-403.

15. Wang Z-X, Sun L-H, Chen A-P: The efficacy of non-pharmacological methods of pain management in school-age children receiving venepuncture in a paediatric department: a randomized controlled trial of audiovisual distraction and routine psychological intervention. Swiss medical weekly 2008, 138(39-40):579-584.

16. Esmaeili K, Sadeghy S, Iranfar S, Abbasi P, Afkary B: The comparison of the effect of music and rhythmic breathing techniques on pain severity of intravenous cannulation during blood transfusion. Journal of Kermanshah University of Medical Sciences (J Kermanshah Univ Med Sci) 2008, 12(2).

17. Shahabi $M$, Kalani D, Eghbal M, Alawi H, Abedsaeidi J: Comparison of the effect of EMLA anesthetic ointment a (EMLA) and the distraction of music on pain in school-age children the hospital vessel Ayatollah dastgheib Shahid Allah (RA) in Shiraz. nursing and midwifery college publication 2006, 16(56):8-12.

18. Sadeghi T, Nayshabori M, Solaimani M, Bahrami N: The effect of swelling the balloon on the pain caused by intravenous way children open. Scientific journal of Kurdistan University of medical sciences, Qazvin 2010, 14(3):68-72.

19. bagherian, borhani, abaszadeh: The effect of non druge on pain control methods of alleviating the pain caused by the vessel in school-age children admitted to the Centre of Thalassemia in Kerman. Journal of the Faculty of nursing and midwifery in uremia 2013, 10(6):0-0.

20. Rostami M, Abazari F, Pouraboli B, Jahani U, Shirzadi F: The effects of massage on pain severity with Hugo point of ice in the veins of children with Thalassemia in the specialized center of medicine, Kerman SAMEN alhojaj. Journal of surgical nursing 2014, 3(3):157-162.

21. Bakhshi F, Eslami M, Younesi M: Therapeutic effect of colors of green, yellow and Red a review article. Abstracts of the first National Conference on applied research in public health and sustainable development 2013, 1(1).

22. Voepel-Lewis T, Merkel S, Tait AR, Trzcinka A, Malviya S: The reliability and validity of the Face, Legs, Activity, Cry, Consolability observational tool as a measure of pain in children with cognitive impairment. Anesthesia \& Analgesia 2002, 95(5):1224-1229. 
23. Alhani $F$, Shad $H$, Anoosheh M, Hajizadeh $E$ : The effect of programmed distraction on the pain caused by venipuncture among adolescents on hemodialysis. Pain Management Nursing 2010, 11(2):85-91.

24. Nikfarid L, GHAMAR, YOUSEFI R, Namazian M, Namdar F, AZAM, NEZAMI M: Comparison of EMLA cream versus local refrigeration for reducing venipuncture-related pain in pediatric patients of Children's Medical Center, 2008. 2010.

25. Abuelkheir M, Alsourani D, Al-Eyadhy A, Temsah M, Meo S, Alzamil F: EMLA ${ }^{\circledR}$ cream: A painrelieving strategy for childhood vaccination. Journal of International Medical Research 2014:0300060513509473.

26. Pormovahed Z, Salimi T, Dehghani K, Yasini M, Shakiba M, Tavangar H, Shahri T: Comparison of two anesthesia methods of music and cream emla on the severity of the pain of the vessel in children. Journal of school of nursing and midwifery, Iran University of medical sciences 2008, 21(55):47-53.

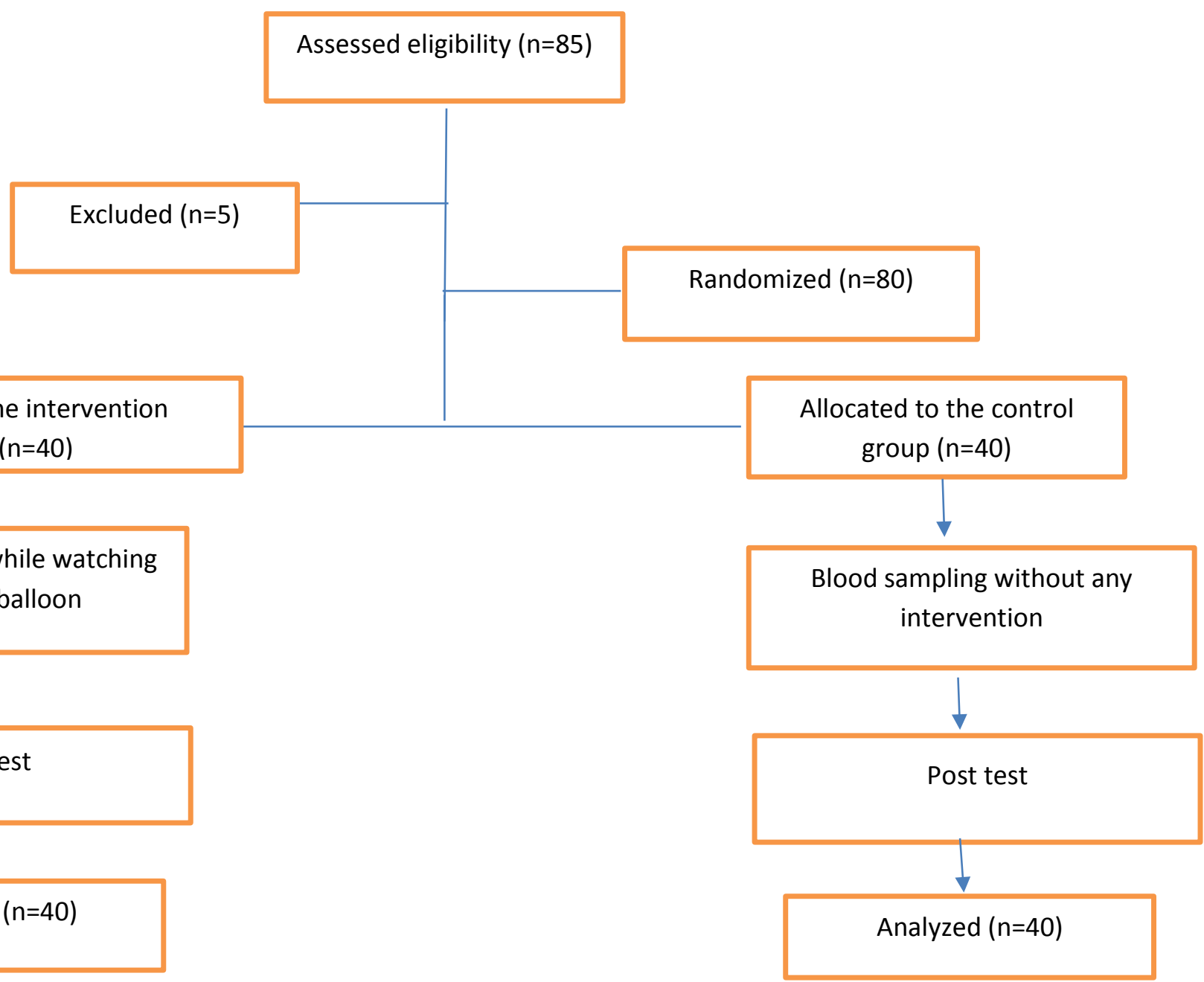

Figure 1: The steps taken to conduct the study 
Table 1: The participants' Quantitative demographic data in the intervention and control groups

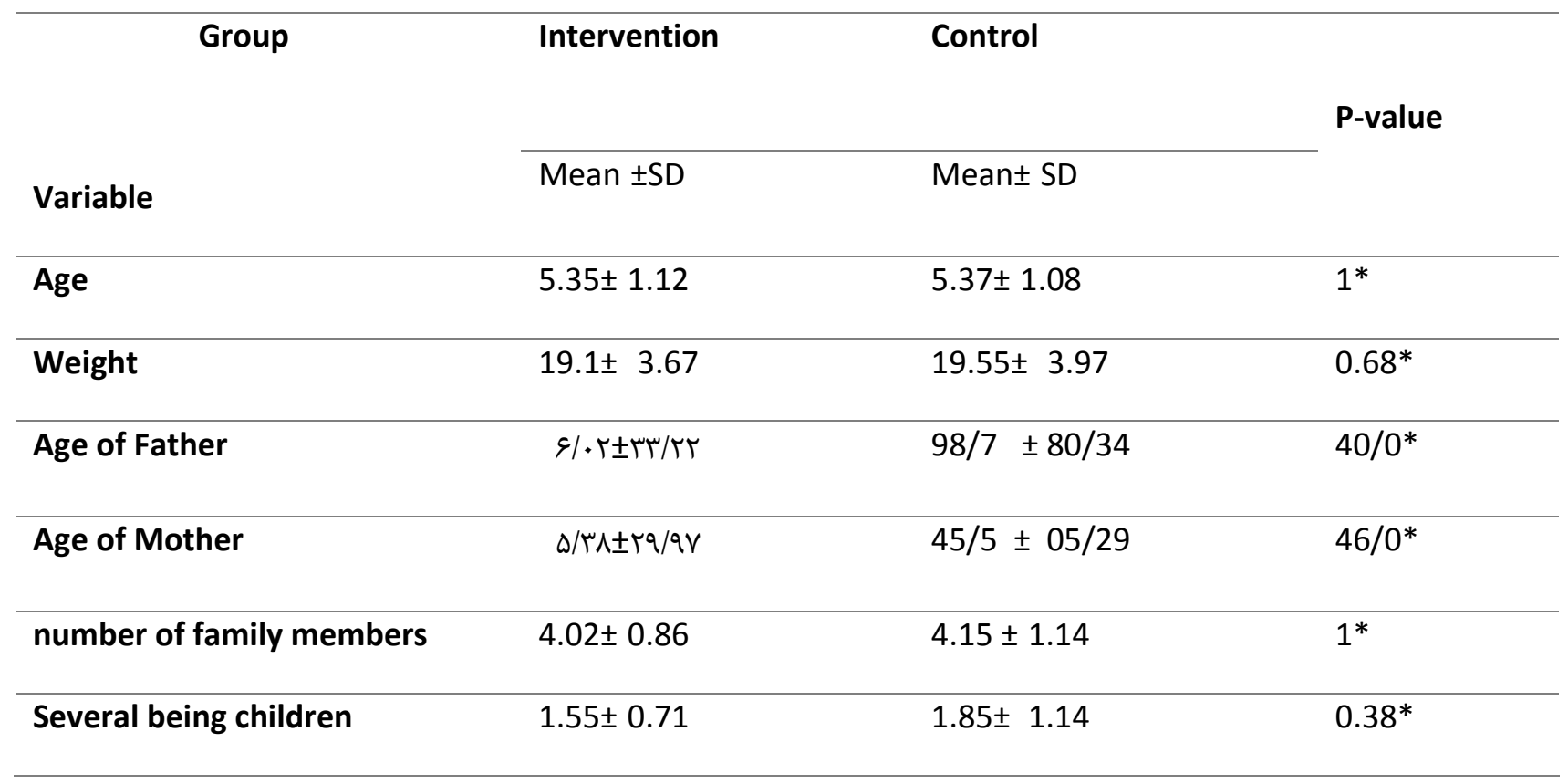

SD, standard deviation.

*t Test 
Table 2: The participants' Qualitative demographic data in the intervention and control groups

\begin{tabular}{|c|c|c|c|}
\hline Group variable & $\begin{array}{l}\text { intervention } \\
\text { n }(\%)\end{array}$ & $\begin{array}{l}\text { control } \\
\text { n }(\%)\end{array}$ & $p$ - value \\
\hline $\operatorname{sex}$ & & & $* 0.34$ \\
\hline Girl & $18(45)$ & $15(37.5)$ & \\
\hline Son & $22(55)$ & $25(62.5)$ & \\
\hline education of father & & & $* 0 . r \mu$ \\
\hline diploma and above & $11(27.5)$ & $15(37.5)$ & \\
\hline under diploma & $29(72.5)$ & $25(62.5)$ & \\
\hline education of mother & & & $* 0.12$ \\
\hline diploma and above & $7(17.5)$ & $13(32.5)$ & \\
\hline under diploma & $33(82.5)$ & $27(67.5)$ & \\
\hline father's job & & & $* 0.47$ \\
\hline employee & $9(22.5)$ & $12(30)$ & \\
\hline worker & $5(12.5)$ & $7(17.5)$ & \\
\hline free & $22(55)$ & $15(37.5)$ & \\
\hline unemployed & $4(10)$ & $6(15)$ & \\
\hline mother's job & & & $* 0.35$ \\
\hline housewife & $20(50)$ & $24(60)$ & \\
\hline employee & $10(25)$ & $5(12.5)$ & \\
\hline free & $10(25)$ & $11(27.5)$ & \\
\hline
\end{tabular}

*chi square test 
Table 3: The mean pain scores two group, before and after intervention

variable

pain scores

group

inflating a balloon

independent samples t-

test

p-value

*paired t-test

$* *$ independent $\mathrm{t}$-test

$* * 0.7$ p-value

\begin{tabular}{|c|c|}
\hline Pre -intervention & After-intervention \\
\hline Mean \pm SD & Mean \pm SD \\
\hline $3.32 \pm 1.28$ & $2.65 \pm 1.38$ \\
\hline 2. $87 \pm 1.18$ & $4.2 \pm 1.58$ \\
\hline
\end{tabular}

$* * 0.0001$ 


\section{Supplementary Files}

This is a list of supplementary files associated with this preprint. Click to download.

- tables.pdf

- Newfolder2.zip

- Newfolder2.zip 